\title{
Equilibrium orbit analysis in a free-electron laser with a coaxial wiggler
}

\author{
B. Maraghechi ${ }^{a, b}$, B. Farrokhi ${ }^{a}$, J. E. Willett ${ }^{c}$, and U.-H. Hwang ${ }^{d}$ \\ ${ }^{a}$ Institute for Studies in Theoretical Physics and Mathematics, \\ P.O. Box 19395-5531, Tehran, Iran. \\ ${ }^{b}$ Department of Physics, Amir Kabir University, Tehran, Iran. \\ ${ }^{c}$ Department of Physics and Astronomy, University of \\ Missouri-Columbia, Columbia, Missouri 65211. \\ ${ }^{d}$ Physics Department, Korea University of Technology and Education, Chunan, Choongnam \\ 330-860, Korea
}

PACs number(s): 41.60.cr, 52.75.Ms

\begin{abstract}
An analysis of single-electron orbits in combined coaxial wiggler and axial guide magnetic fields is presented. Solutions of the equations of motion are developed in a form convenient for computing orbital velocity components and trajectories in the radially dependent wiggler. Simple analytical solutions are obtained in the radially-uniform-wiggler approximation and a formula for the derivative of the axial velocity $v_{\|}$with respect to Lorentz factor $\gamma$ is derived. Results of numerical computations are presented and the characteristics of the equilibrium orbits are discussed. The third spatial harmonic of the coaxial wiggler field gives rise to group $I I I$ orbits which are characterized by a strong negative mass regime.
\end{abstract}




\section{INTRODUCTION}

Most free-electron lasers employ a wiggler with either a helically symmetric magnetic field generated by bifilar current windings or a linearly symmetric magnetic field generated by alternating stacks of permanent magnets. A uniform static guide magnetic field is also frequently employed. Single-particle orbits in these helical and planar fields combined with an axial guide field have been analyzed in detail and have played a role in the development of

free-electron lasers [1]. Harmonics of gyroresonance for off-axis electrons caused by the radial variation of the magnetic field of a helical wiggler is found by Chu and Lin [2]. Recently the feasibility of using a coaxial wiggler in a free-electron laser has been investigated. Freund et al. 3, 34 studied the performance of a coaxial hybrid iron wiggler consisting of a central rod and a coaxial ring of alternating ferrite and dielectric spacers inserted in a uniform static axial magnetic field. McDermott et al. [5] proposed the use of a wiggler consisting of a coaxial periodic permanent magnet and transmission line. Coaxial devices offer the possibility of generating higher power than conventional free-electron lasers and with a reduction in the beam energy required to generate radiation of a given wavelength.

In the present paper, single-particle orbits in a coaxial wiggler are studied. The wiggler magnetic field is radially dependent with the fundamental plus the third spatial harmonic component and a uniform static axial magnetic field present. In Sec. II the scalar equations of motion are introduced and reduced to a form which is correct to first order in the wiggler field. In Sec. III solutions of the equations of motion are developed in a form suitable for computing the electron orbital velocity and trajectory in the radially dependent magnetic field of a coaxial wiggler. The special case of a radially independent wiggler is also analyzed. In Sec. IV the results of numerical computations of the wiggler field components, velocity components, radial excursions, and the $\Phi$ function for locating negative mass regimes are presented and discussed. In Sec. V some conclusions are presented. 


\section{EQUATIONS OF MOTION}

Electron motions in a static magnetic field $\mathbf{B}$ may be determined by solution of the vector equation of motion

$$
\frac{d \mathbf{v}}{d t}=\frac{-e}{\gamma m c} \mathbf{v} \times \mathbf{B}
$$

where $\mathbf{v},-e$, and $m$ are the velocity, charge, and (rest) mass, respectively, of the electron. Lorentz factor $\gamma$ is a constant given by

$$
\gamma=\left(1-v^{2} / c^{2}\right)^{-1 / 2}
$$

where $v=|\mathbf{v}|$ is the constant electron speed.

The total magnetic field inside a coaxial wiggler will be taken to be of the form

$$
\begin{aligned}
& \mathbf{B}=B_{r} \hat{\mathbf{r}}+B_{z} \hat{\mathbf{z}}, \\
& B_{r}=B_{w} F_{r}(r, z), \\
& B_{z}=B_{0}+B_{w} F_{z}(r, z),
\end{aligned}
$$

where $B_{0}$ is a uniform static axial guide field, and $F_{r}$ and $F_{z}$ are known functions of cylindrical coordinates $r$ and $z$. Equation (1) may be written in the scalar form

$$
\begin{aligned}
& \frac{d v_{r}}{d t}-\frac{v_{\theta}^{2}}{r}=-v_{\theta}\left(\Omega_{0}+\Omega_{w} F_{z}\right), \\
& \frac{d v_{\theta}}{d t}+\frac{v_{\theta} v_{r}}{r}=v_{r}\left(\Omega_{0}+\Omega_{w} F_{z}\right)-v_{z} \Omega_{w} F_{r}, \\
& \frac{d v_{z}}{d t}=v_{\theta} \Omega_{w} F_{r}
\end{aligned}
$$

$\Omega_{0}$ and $\Omega_{w}$ are relativistic cyclotron frequencies given by

$$
\begin{aligned}
& \Omega_{0}=\frac{e B_{0}}{\gamma m c}, \\
& \Omega_{w}=\frac{e B_{w}}{\gamma m c} .
\end{aligned}
$$

Initial conditions will be chosen such that the transverse motion of the electron in the $B_{0}$ field vanishes in the limit as $B_{w}$ approaches zero. Then, in order to develop a solution to first order in the wiggler field $B_{w}$, the scalar equations of motion will be approximated by 


$$
\begin{aligned}
\frac{d v_{r}}{d t} & =-\Omega_{0} v_{\theta}, \\
\frac{d v_{\theta}}{d t} & =\Omega_{0} v_{r}-v_{\|} \Omega_{w} F_{r}, \\
\frac{d v_{z}}{d t} & =0 .
\end{aligned}
$$

with the wiggler field approximated by the fundamental plus the third spatial harmonic component,

$$
F_{r}=F_{r 1} \sin \left(k_{w} z\right)+F_{r 3} \sin \left(3 k_{w} z\right)
$$

where

$$
\begin{aligned}
F_{r n} & \equiv G_{n}^{-1}\left[S_{n} I_{1}\left(n k_{w} r\right)+T_{n} K_{1}\left(n k_{w} r\right)\right] \\
G_{n} & \equiv I_{0}\left(n k_{w} R_{\text {out }}\right) K_{0}\left(n k_{w} R_{\text {in }}\right)-I_{0}\left(n k_{w} R_{\text {in }}\right) K_{0}\left(n k_{w} R_{\text {out }}\right), \\
S_{n} & \equiv \frac{2}{n \pi} \sin \left(\frac{n \pi}{2}\right)\left[K_{0}\left(n k_{w} R_{\text {in }}\right)+K_{0}\left(n k_{w} R_{\text {out }}\right)\right] \\
T_{n} & \equiv \frac{2}{n \pi} \sin \left(\frac{n \pi}{2}\right)\left[I_{0}\left(n k_{w} R_{\text {in }}\right)+I_{0}\left(n k_{w} R_{\text {out }}\right)\right]
\end{aligned}
$$

and $n=1,3 ; R_{\text {in }}$ and $R_{\text {out }}$ are the inner and outer radii of the coaxial waveguide, $k_{w}=2 \pi / \lambda_{w}$ where $\lambda_{w}$ is the wiggler (spatial) period, and $I_{0}, I_{1}, K_{0}$, and $K_{1}$ are modified Bessel functions.

\section{ORBITAL ANALYSIS}

\section{A. Radially dependent wiggler}

The scalar equations of motion may be solved to determine the electron orbital velocity and trajectory in a coaxial wiggler. Equation (13) yields

$$
v_{z}=v_{\|}
$$

where the constant $v_{\|}$is the root-mean-square axial velocity component. With the initial axial position taken to be $z_{0}=0$,

$$
z=v_{\|} t
$$


Equations (11), (12), (14), and (20) may be combined to obtain

$$
\frac{d^{2} v_{r}}{d t^{2}}+\Omega_{0}^{2} v_{r}=f(t)
$$

where

$$
f(t)=\Omega_{0} \Omega_{w} v_{\|}\left[F_{r 1} \sin \left(k_{w} v_{\|} t\right)+F_{r 3} \sin \left(3 k_{w} v_{\|} t\right)\right]
$$

By the method of variation of parameters, a solution of Eq. (21) may be obtained in the form

$$
\begin{aligned}
v_{r}= & {\left[-v_{\theta 0}+\Omega_{0}^{-1} \int_{0}^{t} f(\tau) \cos \left(\Omega_{0} \tau\right) d \tau\right] \sin \left(\Omega_{0} t\right) } \\
& +\left[v_{r 0}-\Omega_{0}^{-1} \int_{0}^{t} f(\tau) \sin \left(\Omega_{0} \tau\right) d \tau\right] \cos \left(\Omega_{0} t\right)
\end{aligned}
$$

where $v_{r 0}$ and $v_{\theta 0}$ are initial radial and azimuthal velocity components. Then Eq. (11) yields

$$
\begin{aligned}
v_{\theta} & =\left[v_{\theta 0}-\Omega_{0}^{-1} \int_{0}^{t} f(\tau) \cos \left(\Omega_{0} \tau\right) d \tau\right] \cos \left(\Omega_{0} t\right) \\
& +\left[v_{r 0}-\Omega_{0}^{-1} \int_{0}^{t} f(\tau) \sin \left(\Omega_{0} \tau\right) d \tau\right] \sin \left(\Omega_{0} t\right) .
\end{aligned}
$$

The orbital velocity is given to first order in $B_{w}$ by Eqs. (23), (24), and (19). The trajectory may then be computed using

$$
\begin{aligned}
& r=r_{0}+\int_{0}^{t} v_{r}(\tau) d \tau, \\
& \theta=\theta_{0}+\int_{0}^{t} v_{\theta}(\tau) d \tau
\end{aligned}
$$

and Eq. (20).

\section{B. Radially uniform wiggler}

By neglecting the radial variation of $F_{r 1}$ and $F_{r 3}$, a solution of Eq. (21) may be obtained in the form

$$
v_{r}=\alpha_{1} \sin \left(k_{w} v_{\|} t\right)+\alpha_{3} \sin \left(3 k_{w} v_{\|} t\right)
$$

where 


$$
\alpha_{n}=\frac{\Omega_{0} \Omega_{w} v_{\|} F_{r n}}{\Omega_{0}^{2}-n^{2} k_{w}^{2} v_{\|}^{2}} \quad(n=1,3) .
$$

Equation (11) then yields

$$
v_{\theta}=-\Omega_{0}^{-1} k_{w} v_{\|} \alpha_{1} \cos \left(k_{w} v_{\|} t\right)-\Omega_{0}^{-1}\left(3 k_{w} v_{\|} \alpha_{3}\right) \cos \left(3 k_{w} v_{\|} t\right) .
$$

The corresponding initial conditions are

$$
\begin{gathered}
v_{r 0}=0 \\
v_{\theta 0}=-\Omega_{0}^{-1} k_{w} v_{\|} \alpha_{1}-\Omega_{0}^{-1}\left(3 k_{w} v_{\|} \alpha_{3}\right) .
\end{gathered}
$$

Root-mean-square values of the velocity components may be determined by use of Eqs. (27), (28), and (19). Replacing $v^{2}$ by its root-mean-square value in Eq. (2) then yields

$$
\frac{v_{\|}^{2}}{c^{2}}\left[1+\frac{1}{2}\left(\frac{\alpha_{1}}{v_{\|}}\right)^{2}+\frac{1}{2} \Omega_{0}^{-2} k_{w}^{2} \alpha_{1}^{2}+\frac{1}{2}\left(\frac{\alpha_{3}}{v_{\|}}\right)^{2}+\frac{9}{2} \Omega_{0}^{-2} k_{w}^{2} \alpha_{3}^{2}\right]=1-\gamma^{-2} .
$$

The derivative of $v_{\|}$with respect to $\gamma$ may be obtained from Eq. (32) and, after some algebra, cast into the form

$$
\frac{d v_{\|}}{d \gamma}=\frac{c^{2}}{\gamma \gamma_{\|}^{2} v_{\|}} \Phi
$$

where

$$
\Phi=1-\frac{\sum_{n=1,3}\left(\Omega_{0}^{2}-n^{2} k_{w}^{2} v_{\|}^{2}\right)^{-3} \gamma_{\|}^{2} \Omega_{w}^{2} F_{r n}^{2} \Omega_{0}^{2}\left(\Omega_{0}^{2}+3 n^{2} k_{w}^{2} v_{\|}^{2}\right)}{2+\sum_{n=1,3}\left(\Omega_{0}^{2}-n^{2} k_{w}^{2} v_{\|}^{2}\right)^{-3} \Omega_{w}^{2} F_{r n}^{2} \Omega_{0}^{2}\left(\Omega_{0}^{2}+3 n^{2} k_{w}^{2} v_{\|}^{2}\right)}
$$

This equation may be used to establish the existence of a negative mass regime.

\section{NUMERICAL RESULTS}

A numerical computation is conducted to investigate the properties of the equilibrium orbits of electrons inside a coaxial wiggler. Wiggler wavelength $2 \pi / k_{w}$ and lab-frame electron density $n_{0}$ were taken to be $3 \mathrm{~cm}$ and $10^{12} \mathrm{~cm}^{-3}$, respectively. The wiggler magnetic field $B_{w}$ was taken to be $3745 \mathrm{G}$ which corresponds to the relativistic wiggler frequency $\Omega_{w} / c k_{w}=$ 
0.442. Electron-beam energy $(\gamma-1) m_{0} c^{2}$ was taken to be $700 \mathrm{keV}$, corresponding to a Lorentz factor $\gamma=2.37$. The axial magnetic field $B_{0}$ was varied from 0 to $25.3 \mathrm{kG}$, corresponding to a variation from 0 to 3 in the normalized relativistic cyclotron frequency $\Omega_{0} / c k_{w}$ associated with $B_{0}$. The inner and outer radii of the coaxial wiggler were assumed to be $R_{i n}=1.5 \mathrm{~cm}$ and $R_{\text {out }}=3 \mathrm{~cm}$, respectively.

Figure 1 shows the variation of the axial velocity of the quasi-steady-state orbits with the axial guide magnetic field for three classes of solutions. Group I orbits for which $0<$ $\Omega_{0}<k_{w} v_{\|}$, group II orbits with $k_{w} v_{\|}<\Omega_{0}<3 k_{w} v_{\|}$, and group III orbits with $\Omega_{0}>$ $3 k_{w} v_{\|}$. Existence of group III orbits is due to the presence of the third spatial harmonic of the wiggler field, which also produces the second magnetoresonance at $\Omega_{0} \approx 3 k_{w} v_{\|}$. The narrow width of the second resonance at $\Omega_{0} / c k_{w} \approx 2.7$ compared to the width of the first magnetoresonance at $\Omega_{0} \approx k_{w} v_{\|}$is illustrated in Fig. 1B. This is due to the relatively weak third harmonic compared to the fundamental component of the wiggler field. It should be noted that although the exact resonances $\Omega_{0}=k_{w} v_{\|}$and $\Omega_{0}=3 k_{w} v_{\|}$occur at the origin where $v_{\|} / c=\Omega_{0} / c k_{w}=0$, the first "magnetoresonance" in the literature refers to the group II orbits with the cyclotron frequencies around $\Omega_{0} / c k_{w} \approx 1$ in Fig. 1 . Similarly we refer to the group III orbits with the cyclotron frequency around $\Omega_{0} / c k_{w} \approx 2.7$ as the second magnetoresonance.

The rate of change of the electron axial velocity with electron energy is proportional to $\Phi$ and is equal to unity in the absence of the wiggler field. Figure 2 illustrates the dependence of $\Phi$ on the radial wiggler magnetic field and the axial guide magnetic field $B_{0}$. The curves corresponding to the group I and II orbits are almost unaffected by the third harmonic and are almost the same as in Ref. [6] where the third harmonic is neglected. A negative mass regime (i.e., negative $\Phi$ for which a decrease in the axial velocity results in an increase in the electron energy) is found for group III orbits which is stronger than that of the group II orbits.

Equations (15)-(18) are used to calculate the radial components of the wiggler field $F_{r 1}$ and $F_{r 3}$. For the axial component the following expressions are used [4] 


$$
\begin{gathered}
F_{z}=F_{z 1} \cos \left(k_{w} z\right)+F_{z 3} \cos \left(3 k_{w} z\right), \\
F_{z n}=G_{n}^{-1}\left[S_{n} I_{0}\left(n k_{w} r\right)-T_{n} K_{0}\left(n k_{w} r\right)\right] .
\end{gathered}
$$

Figure 3 shows the variation of the amplitudes of the wiggler magnetic field (divided by $B_{w}=3745 \mathrm{G}$ ) with radius, for the first and third spatial harmonics. For the first harmonic the radial component, $F_{r 1}$, has a minimum at $r \approx 2.28 \mathrm{~cm}$ and the axial component, $F_{z 1}$, changes sign around this point. Mc Dermott et al. [5] have demonstrated the stability of a thin annular electron beam when $F_{r 1}$ is minimum at the beam radius. The radial and axial components of the third harmonic of the wiggler $F_{r 3}$ and $F_{z 3}$ are also shown in Fig. 3 . Magnitudes of $F_{r 3}$ and $F_{z 3}$ both are minimum at $r \approx 2.28 \mathrm{~cm}$ where $F_{r 1}$ is minimum. This is actually an inflection point for $F_{z 3}$.

Variations of the radial components of the first and third spatial harmonics of the normalized wiggler magnetic field $F_{r 1}$ and $F_{r 3}$ with the wiggler wave number $k_{w}$ are shown in Fig. 4. Figure 4 also shows the dimensionless transverse velocity coefficients $\bar{\alpha}_{1}=\alpha_{1} / c$ and $\bar{\alpha}_{3}=\alpha_{3} / c$ for the initial orbit radius $r_{0} \approx 2.28 \mathrm{~cm}$ where $F_{r 1}$ is minimum. The cyclotron frequency $\Omega_{0} / c k_{w} \approx 2.7$ is taken at the second magnetoresonance, and our choice of $3 \mathrm{~cm}$ for the wiggler wavelength corresponds to $k_{w} \approx 2.1 \mathrm{~cm}^{-1}$. It can be observed that at this wave number although the radial component of the wiggler field at the first harmonic $F_{r 1}$ is much larger than the third harmonic $F_{r 3}$, the transverse velocity coefficients of the third harmonic $\bar{\alpha}_{3}$ are larger than $\bar{\alpha}_{1}$. This shows that the third harmonic may have considerable effects around the second magnetoresonance at $\Omega_{0} \approx 3 k_{w} v_{\|}$. Away from this resonance Eq. (28) shows that $\alpha_{3}$ will be of the order of $F_{r 3}$.

In order to study the transverse motion of electrons in the radially dependent wiggler field Eqs. (11), (12), (25), and (26) are solved numerically with the initial conditions chosen so that, in the limit of zero wiggler field, there is axial motion at constant velocity $v_{\|}$but no Larmor motion. Figure 5 shows the variation of the radial and azimuthal components of electron velocity with $z\left(=v_{\|} t\right)$. The normalized cyclotron frequency $\Omega_{0} / c k_{w}$ is chosen to be 0.5, 1.2, and 3 for group I, II, and III orbits, respectively, which are somewhat away from the 
magnetoresonances. Solid curves correspond to the initial orbit radius $r_{0}=2.28 \mathrm{~cm}$, which is at the point where $F_{r 1}$ is minimum. Broken curves correspond to $r_{0}=1.8 \mathrm{~cm}$, which is away from the $F_{r 1}$ minimum. It can be observed that the spatial periodicity of $v_{r}$ and $v_{\theta}$ for the first two groups is equal to one wiggler wavelength, which is the same as that of the first harmonic. Although group III orbits have a clear sinusoidal shape at $r_{0}=2.28 \mathrm{~cm}$ ( solid curves), the slight deviations from sinusoidal shape are obvious at $r_{0}=1.8 \mathrm{~cm}$ (broken curves). This is because that at $r_{0}=2.28 \mathrm{~cm}$ where $F_{r 1}$ is minimum $F_{r 3}$ is very small. Therefore away from the resonance the third harmonic plays almost no role, at $r_{0}=2.28$ $\mathrm{cm}$. Moving away from $F_{r 1}$ minimum to $r_{0}=1.8 \mathrm{~cm}$, however, increases the magnitude of $F_{r 3}$ slightly making the effect of the third harmonic noticeable on group III orbits, which are away from the second magnetoresonance, in Fig. 5.

Figure 6 shows the variations of $v_{r} / c$ and $v_{\theta} / c$ with $z\left(=v_{\|} t\right)$ for group III orbits when the cyclotron frequency is adjusted at the second magnetoresonance at $\Omega_{0} / c k_{w} \approx 2.7$. At $r_{0}=$ $2.28 \mathrm{~cm}$ the periodicity is approximately equal to $\lambda_{w} / 3$, which is the same as that of the third harmonic, and shows the strong influence of the third harmonic on the transverse velocity components. Going away from the $F_{r 1}$ minimum to $r_{0}=1.8 \mathrm{~cm}$ makes the amplitudes of oscillations of $v_{r}$ and $v_{\theta}$ larger. Broken curves correspond to the solutions of Eqs. (27) and (29) when the radial variation of the wiggler field is neglected. These solutions do not differ appreciably from the r-dependent solutions for $r_{0}=2.28 \mathrm{~cm}$ because at $F_{r 1}$ minimum the radial excursions are small for group III orbits. Away from the $F_{r 1}$ minimum at $r_{0}=1.8$ $\mathrm{cm}$, however, deviations are noticeable due to the larger radial excursions.

Figure 7 shows $v_{r} / c$ versus $z$ for group II orbits for r-dependent wiggler (broken curves) and $\mathrm{r}$-independent wiggler (solid curves). The initial orbit radius is taken at $r_{0}=2.28 \mathrm{~cm}$ and the cyclotron frequency is chosen around the first magnetoresonance at $\Omega_{0} / c k_{w}=0.9$. Large radial excursions of electrons for group II orbits make the transverse velocity strongly affected by the radial dependency of the wiggler field. The amplitude is also modulated in space with the wavelength of around $16 \lambda_{w}=48 \mathrm{~cm}$.

The radial excursion $r$ shown in Fig. 8 corresponds to the cyclotron frequencies away 
from the magnetoresonances at $\Omega_{0} / c k_{w}$ equal to $0.5,1.2$, and 3 for the group I, II, and III orbits, respectively. Solid curves correspond to the $r_{0}=2.28 \mathrm{~cm}$ and the broken curves correspond to $r_{0}=1.8 \mathrm{~cm}$. It can be noticed that when the electrons are injected into the wiggler at $r_{0}=2.28 \mathrm{~cm}$, where $F_{r 1}$ is minimum, electron orbits remain well away from the waveguide walls at $R_{\text {in }}=1.5 \mathrm{~cm}$ and $R_{\text {out }}=3 \mathrm{~cm}$.

Figure 9 compares the radial excursions of group III orbits at the second magnetoresonance at $\Omega_{0} / c k_{w}=2.7$, (solid curve) with those slightly away from the resonance at $\Omega_{0} / c k_{w}=3$, (broken curve). Influence of the third harmonic can be clearly seen through the modulation of the third harmonic by the first harmonic when the cyclotron frequency is adjusted at the second magnetoresonance.

\section{CONCLUSIONS}

The third spatial harmonic of the coaxial wiggler field gives rise to the group III orbits with $\Omega_{0}>3 k_{w} v_{\|}$. This relatively weak third harmonic makes the width of the second magnetoresonance narrow compared to the first magnetoresonance. A strong negative mass regime is found for the group III orbits. By adjusting the cyclotron frequency at the second magnetoresonance the wiggler induced velocity of the group III orbits was found to increase considerably. When the electrons are injected into the wiggler where its magnetic field is minimum the electron orbits remain well away from the waveguide boundaries.

Harmonic gyroresonance of electrons in the combined helical wiggler and axial guide magnetic field is reported by Chu and Lin [2]. In their analysis the relativistic single particle equation of motion is used with the axial velocity as well as the axial magnetic field of the wiggler averaged along the axial direction. By assuming near steady-state orbits for off-axis electrons they found that the radial variation of the wiggler magnetic field produces a harmonic structure in the transverse force. This force, in turn, comprises oscillations at all harmonics of $k_{w} z$. It should be noted that there is no harmonic structure in the helical

wiggler itself and the higher velocity harmonics vanish for the exact steady-state orbits of 
the on-axis electrons. Moreover, higher harmonics do not appear in the one dimensional helical wiggler where the radial variation is neglected.

In the present analysis of coaxial wiggler, on the other hand, equation of motion is written to first order in the wiggler amplitude. With this approximation axial component of the wiggler field has no contribution to the problem leaving the axial velocity as a constant. Magnetic field of a coaxial wiggler is composed of a fundamental plus a large number of odd spatial harmonics, which directly appear in the magnetic force represented by $f(t)$ in Eq. (22). Third harmonic in $f(t)$ appears in the transverse velocity components as a part of the integrands in Eqs. (23) and (24) and is also demonstrated numerically for the radially dependent coaxial wiggler, but for the radially uniform wiggler the third harmonic is explicit in the solutions Eqs. (27)-(29). 


\section{REFERENCES}

[1] H. P. Freund and J. M. Antonsen, Jr., Principles of Free-Electron Laser (Chapman and Hall, London, 1996), Chap. 2).

[2] K. R. Chu and A. T. Lin, Phys. Rev. E 67, 3235 (1991).

[3] H. P. Freund, R. H. Jackson, D. E. Pershing, and J. M. Taccetti, Phys. Plasmas 1, 1046 (1994).

[4] H. P. Freund, M. E. Read, R. H. Jackson, D. E. Pershing, and J. M. Taccetti, Phys. Plasmas 2, 1755 (1995).

[5] D. B. McDermott, A. J. Balkcum, R. M. Phillips, and N. C. Luhmann, Jr, Phys. Plasmas 2, $4332(1995)$.

[6] J. E. Willett, U. Hwang, Y. Aktas, and H. Mehdian, Phys. Rev. E 57, 2262 (1998). 


\section{FIGURES}

FIG. 1. Normalized axial velocity $v_{\|} / c$ as a function of the normalized axial-guide magnetic field $\Omega_{0} / c k_{w}$ for group I, II, and III orbits. Narrow width of the second resonance at $\Omega_{0} / c k_{w} \approx 2.7$ compared to the width of the first magnetoresonance at $\Omega_{0} \approx k_{w} v_{\|}$is illustrated in Fig. 1B.

FIG. 2. Factor $\Phi$ as a function of the normalized axial-guide magnetic field $\Omega_{0} / c k_{w}$ for group I, II, and III orbits.

FIG. 3. Radial dependence of the radial and axial magnetic fields (divided by $B_{w}=3745 \mathrm{G}$ ) in the coaxial wiggler for the fundamental and third spatial harmonics.

FIG. 4. Wave-number dependence of the radial components of the normalized wiggler magnetic field $F_{r 1}, F_{r 3}$ and the dimensionless transverse velocity coefficients $\bar{\alpha}_{1}, \bar{\alpha}_{3}$. The normalized cyclotron frequency $\Omega_{0} / c k_{w}=2.7$ is taken at the second magnetoresonance and $r_{0}=2.28 \mathrm{~cm}$.

FIG. 5. Normalized transverse velocity components as a function of axial distance $z$ for the initial orbit radius $r_{0}=2.28 \mathrm{~cm}$ (solid curves) and $r_{0}=1.8 \mathrm{~cm}$ (dotted curves). The normalized cyclotron frequency $\Omega_{0} / c k_{w}$ is $0.5,1.2$, and 3 for the group I, II, and III orbits, respectively.

FIG. 6. Normalized transverse velocity components as a function of axial distance $z$ for group III orbits, at the second magnetoresonance $\Omega_{0} / c k_{w}=2.7$, solid curves correspond to the radial dependent wiggler and broken curves correspond to the radial independent wiggler.

FIG. 7. Normalized radial velocity as a function of axial distance $z$ for group II orbits at the

first magnetoresonance $\Omega_{0} / c k_{w}=0.9$ with $r_{0}=2.28 \mathrm{~cm}$. Solid curves correspond to the radial dependent wiggler and broken curves correspond to the radial independent wiggler.

FIG. 8. Radial excursion $r$ as a function of axial distance $z$ for $r_{0}=2.28 \mathrm{~cm}$ (solid curves) and $r_{0}=1.8 \mathrm{~cm}$ (broken curves) for groups I, II, and III orbits out of resonance at $\Omega_{0} / c k_{w}=0.5,1.2$, and 3 , respectively.

FIG. 9. Radial excursion $r$ as a function of axial distance $z$ for group III orbits at $r_{0}=2.28$ $\mathrm{cm}$. Solid curve corresponds to resonance at $\Omega_{0} / c k_{w}=2.7$ and broken curve corresponds to out of resonance at $\Omega_{0} / c k_{w}=3$. 
Fig. 1
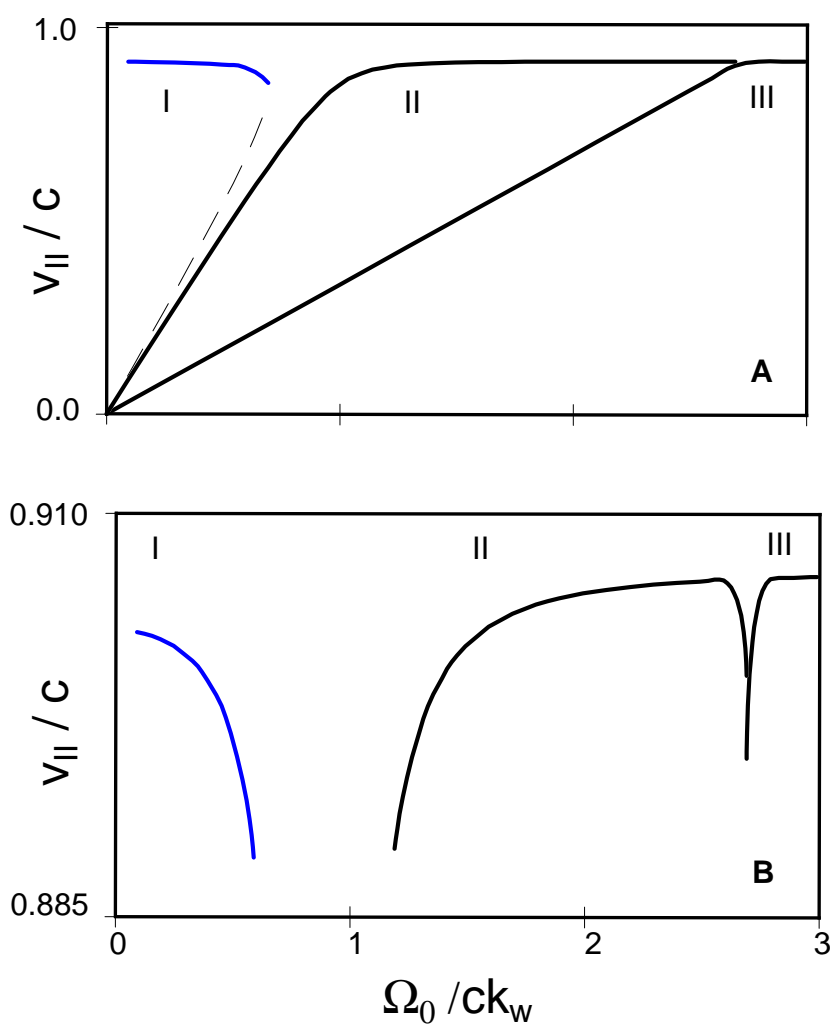
Fig. 2

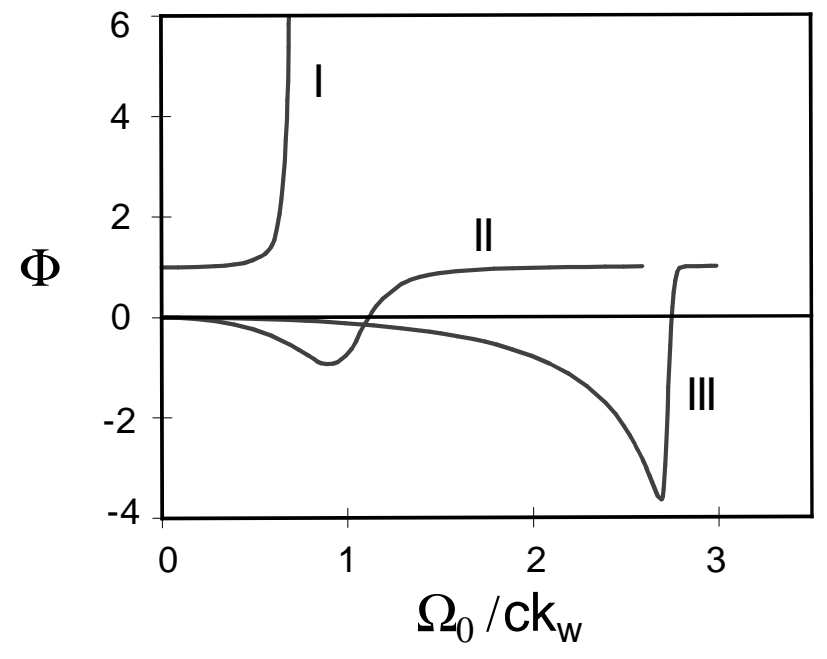


Fig. 3

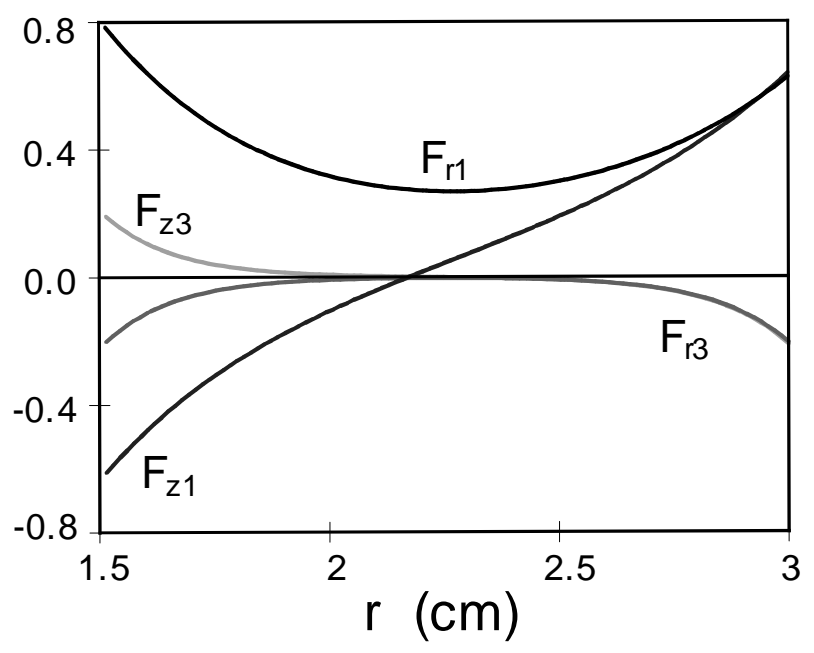


Fig. 4

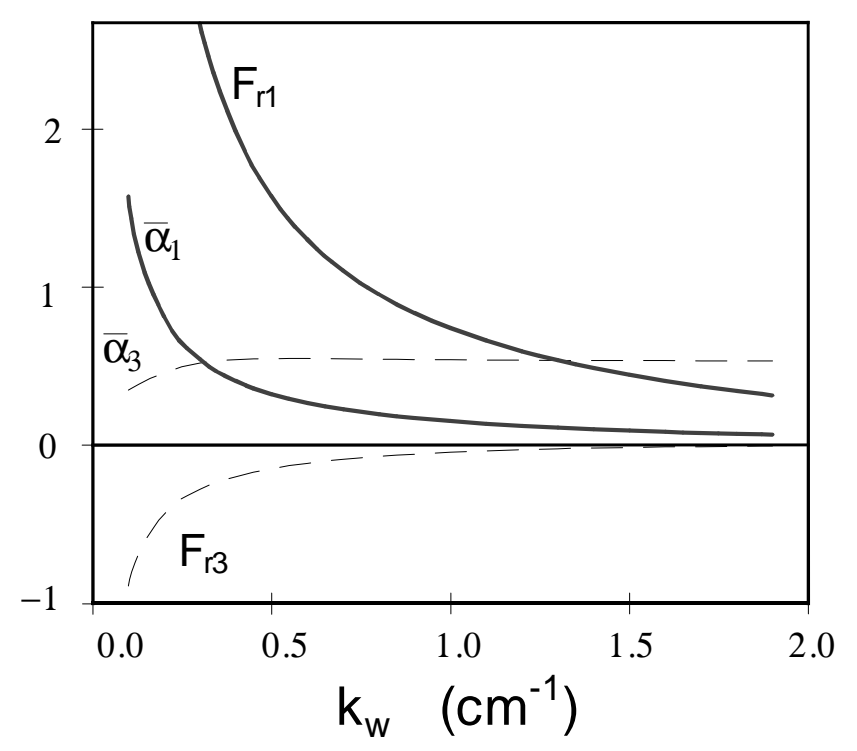


Fig. 5
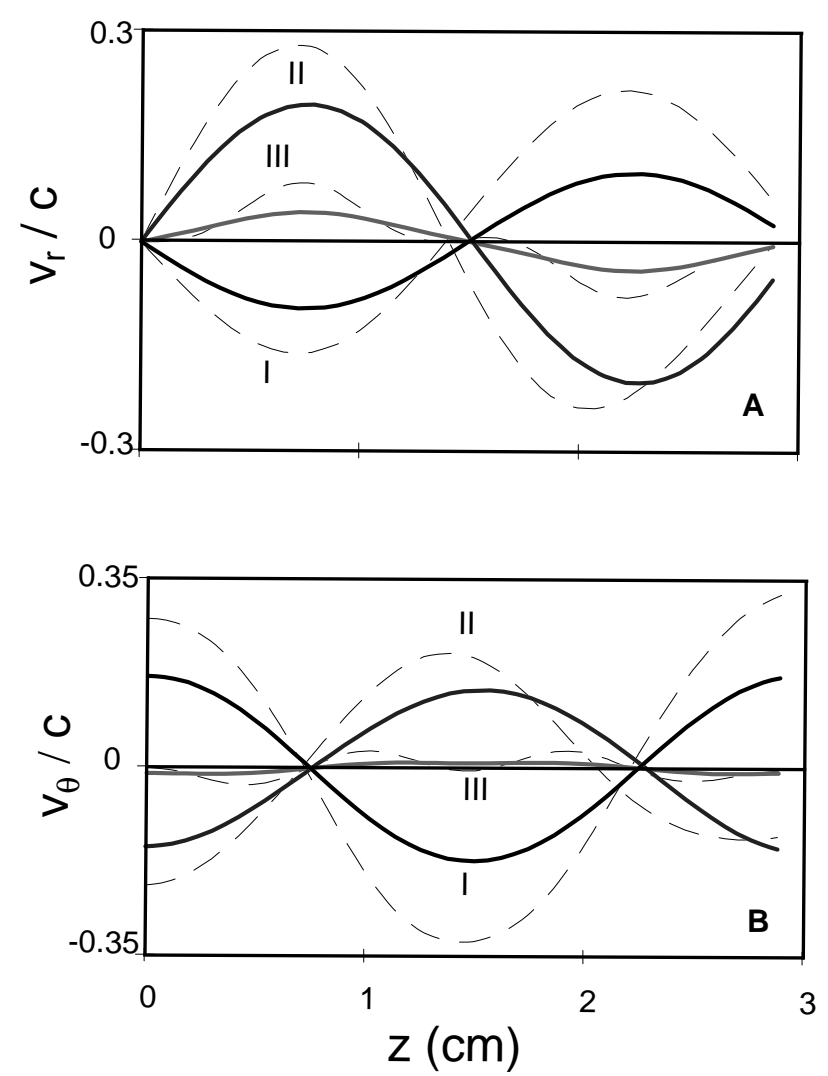
Fig. 6
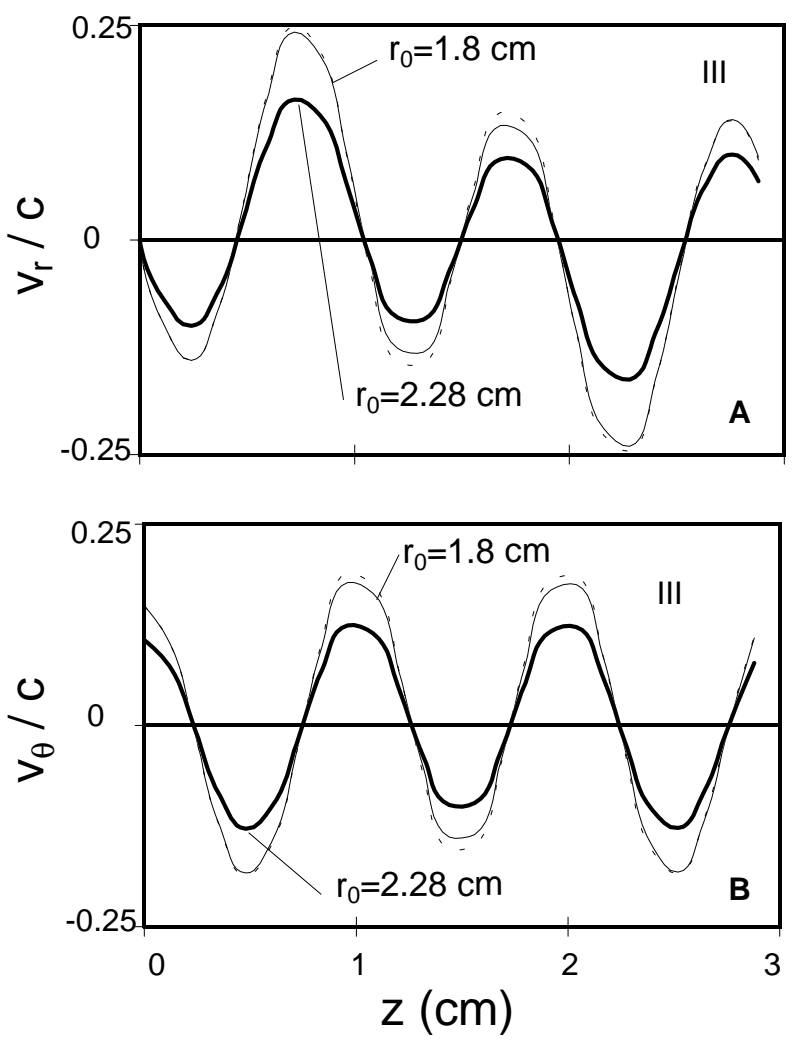
Fig. 7

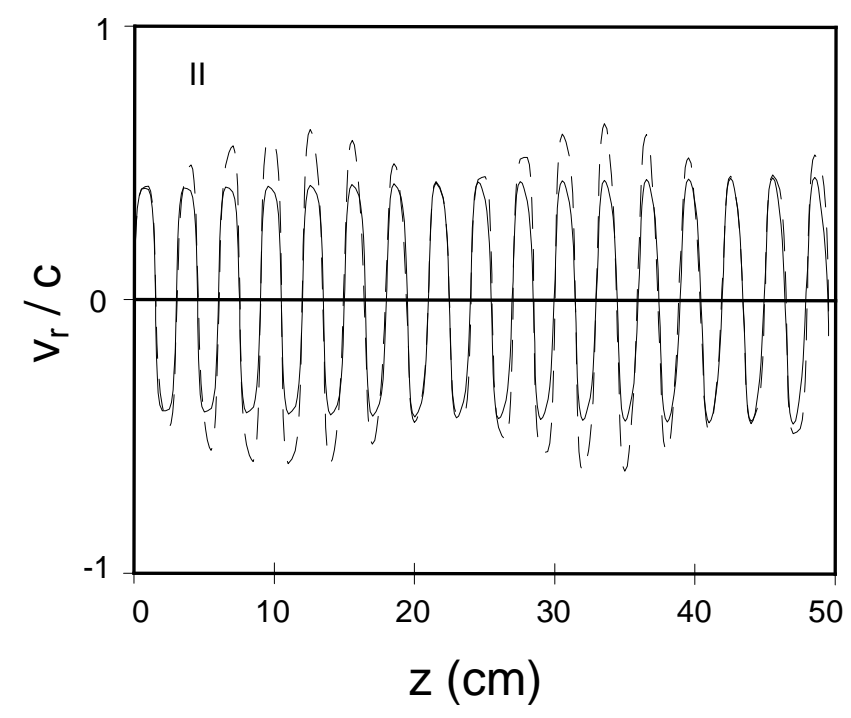


Fig. 8

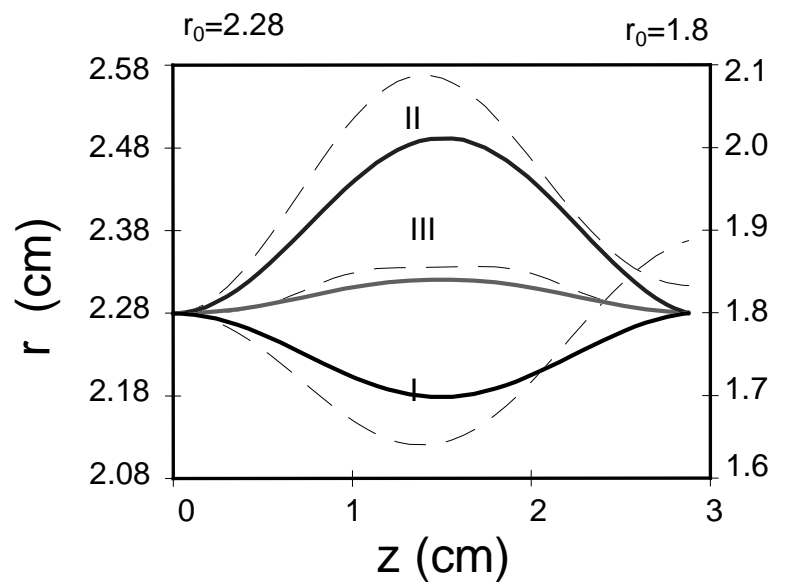


Fig. 9

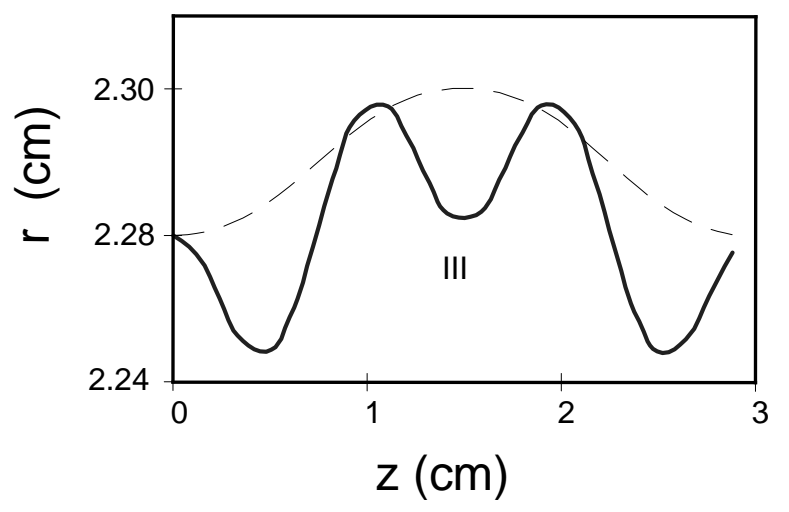

